\title{
SPECTRAL ESTIMATES AND OSCILLATION OF SINGULAR DIFFERENTIAL OPERATORS
}

\author{
WALTER ALLEGRETTO
}

\begin{abstract}
Several estimates are established for the lower spectrum of singular second order elliptic operators. These estimates are obtained by considering the oscillation properties of the operators involved.
\end{abstract}

This paper establishes estimates on the lower spectrum of operators generated by singular elliptic equations. These estimates are obtained by considering the oscillation properties of the operators involved. There is a considerable amount of literature on this subject in the special case where the only singularity comes from the unboundedness of the domain or, equivalently, from the behaviour of the coefficients at a single point. We refer the reader to the books by Glazman [3], Kreith [5], Swanson [12], and the articles by Allegretto [1], Piepenbrink [9], [10], where further references may be found. If the singularity comes from the behaviour of the coefficients on components of the boundary of the domain, the case of interest to us, then there are fewer results. We recall the criteria established some time ago by Kreith [6] for bounded domains and which we shall complement. The spectral properties with which we are concerned are completely determined by the behaviour of the operator near the boundary. An example below will show that due to the error introduced it is in general undesirable to proceed by further localizing our considerations to the behaviour near individual points of the boundary. This fact shows the need to modify the "point" procedures previously employed, and is one of the motivations for our considerations.

Let $E^{m}$ denote Euclidean $m$-space and let $G$ denote a domain in $E^{m}$ with boundary $\partial G$. When $G$ is unbounded we shall always consider the topology of the one point compactification of $G$ in $E^{m}$, so that $\infty \in \partial G$. We let $D_{i}$ denote differentiation with respect to $x_{i}$ for $i=1, \ldots, m$ and introduce in $C_{0}^{\infty}(G)$ the locally uniformly elliptic operator:

$$
l u=-\sum_{i, j=1}^{m} D_{i}\left(a_{i j} D_{j} u\right)+c u .
$$

We shall always assume that the coefficients are real and that: $a_{i j}=a_{j i}$, $a_{i j} \in C^{1}(G), c \in C(G)$. If $l$ is not bounded below then neither will be its selfadjoint extensions and, for the problems we consider, the situation will be

Received by the editors February 13, 1978.

AMS (MOS) subject classifications (1970). Primary 35B05.

Key words and phrases. Elliptic equations, essential spectrum, singular operator, oscillation.

$0002-9939 / 79 / 0000-0011 / \$ 02.50$

(c) 1979 American Mathematical Society 
trivial. Without loss of generality, we therefore assume that $l$ is bounded below and we denote by $L$ its Friedrichs extension. This is the only extension considered in the sequel. If $P$ is any subdomain of $G$ then we denote by $L(P)$ the extension of the operator obtained by restricting $(1)$ to $C_{0}^{\infty}(P)$. We denote by $S(L(P))$ the spectrum of $L(P)$ and by $\mu(P)$ the least point of the essential spectrum $S_{e}(L(P))$. We shall employ oscillation theory to estimate $\mu$ and to determine whether the number of points in $S(L) \cap(-\infty, \mu)$ is infinite. In this connection we state the following definitions which are simple adaptations of well-known ones, [12], [5], [6]. Let $\mathcal{G}$ be a subset of $\partial G$. $L$ oscillates at $\mathcal{G}$ iff given any deleted neighbourhood $U$ of $\mathcal{G}$ there exists a bounded domain $\bar{N} \subset U \cap \bar{G}$ such that $S(L(N)) \cap(-\infty, 0] \neq \varnothing$. We define $k(\mathcal{G})=\sup \{r \mid L$ $-r$ does not oscillate at $\mathcal{G}\}$ to be the oscillation constant of $L$ at $\mathcal{G}$.

We recall [3] that a characteristic sequence $\left(f_{n}\right)$ corresponding to $\lambda \in S_{e}(L)$ is a sequence of normalized functions with no convergent subsequence such that $L\left(f_{n}\right)-\lambda f_{n} \rightarrow 0$. We term a bounded open (in the induced topology) $Z \subset \bar{G}$ regular iff for any $\lambda$ in $S_{e}(L)$ we can modify, by compactness arguments, an associated sequence so that the new sequence vanishes in $Z$. Let $\Re=\bar{G}-\cup(Z \mid Z$ regular). In view of our assumptions, see [3], $\Re \subset$ $\partial G$, with possibly, $\mathscr{T}=\partial G$. The connection between oscillation and spectral theory is given by:

LEMMA 0. (a) $\mu=k(\partial G)=k(\mathfrak{T})$;

(b) $S(L) \cap(-\infty, \mu)$ is infinite iff $L-\mu$ oscillates at $\partial G$.

The proof of Lemma 0 , together with the regularity conditions needed, is either explicitly given in, or follows from, results in [3], [9], [10]. In view of part (a) of the above lemma, we may modify $\partial G$ away from $\mathcal{N}$ in any way, as long as no new singular points are added. Without further reference we shall assume in the sequel that, whenever advantageous, such a perturbation is performed. Even for problems with regular coefficients and smooth boundary, it does not appear to be in general known whether part (b) of the lemma can be replaced by: "( $\left.\mathrm{b}^{\prime}\right) S(L) \cap(-\infty, \mu)$ is infinite iff $L-\mu$ oscillates at $\Re$ ". This can be done in some cases when $\mathfrak{T}$ is a disconnected part of $\partial G$, and analogous problems. The details are given in [10]. This situation is another reason why we consider oscillation at $\partial G$ as opposed to oscillation at only parts of $\partial G$.

It is also a consequence of the above mentioned results that if $x \in \partial G$ then $\mu \leqslant k((x))$. That this gives in general only a very crude upper bound is shown by the following example: Assume that $G \subset E^{2} \cap\left(\left(x_{1}, x_{2}\right) \mid x_{1}>0\right)$ is bounded with regular boundary and that $\left(\left(0, x_{2}\right) \mid-2 \pi<x_{2}<2 \pi\right) \subset \partial G$. Assume that in the strip $\left(\left(x_{1}, x_{2}\right) \mid 0 \leqslant x_{2} \leqslant \pi\right) \cap G, l$ has the form: $-D_{1}\left(x_{1}^{2} D_{1} u\right)-D_{2} D_{2} u$, with the coefficients suitably continued outside the strip. It is easy to see that $k((x))=\infty$ for any $x \in \bar{G}$, but that $\mu \leqslant$ $k\left\{\left(\left(0, x_{2}\right) \mid 0 \leqslant x_{2} \leqslant \pi\right)\right\} \leqslant \frac{5}{4}$. 
Finally we observe that if $\mathscr{T}$ can be decomposed into a finite number of closed mutually disjoint components $\left(\mathscr{T}_{i}\right)$, then $\mu=\inf _{i} k\left(\mathscr{T}_{i}\right)$. It follows that to locate $\mu$ each $\Re_{i}$ can be considered independently of the other parts. We therefore assume that $\Re$ consists of a single component and state our results for this special case.

We next make the following fundamental assumption about $G$ : we assume that there exists a positive regular function $\rho$ from a deleted neighbourhood of $\partial G$ (in the induced topology on $\bar{G}$ ) to $E^{1}$ such that the equipotentials $\rho(x)=a$ are nested closed bounded smooth surfaces for $a$ large and that, for $\beta$ large, $\left\{\cup_{\zeta>\beta}(x \mid \rho(x)=\zeta)\right\}_{\beta}$ gives a deleted neighbourhood base for $\partial G$ in the induced topology. For $G$ regular and bounded we could for example choose $\rho(x)=(\operatorname{dist}(x, \partial G))^{-1}$, but such functions can also be constructed for unbounded domains. This approach has the advantage that once such a $\rho$ is constructed then no further specification of the nature of $\mathscr{T}$ is necessary. It does suffer, however, from the need to know explicitly $\rho$ in order to obtain calculable criteria.

THEOREM 1. Assume that the ordinary differential equation:

$$
-\left(a f^{\prime}\right)^{\prime}+b f=0
$$

oscillates $($ at $\infty)$, where:

$$
\begin{gathered}
a(t)=\int_{\rho(x)=t} \sum a_{i j}(x) D_{i} \rho D_{j} \rho \frac{d S}{|\operatorname{grad} \rho|} ; \\
b(t)=\int_{\rho(x)=t}(c(x)-k) \frac{d S}{|\operatorname{grad} \rho|} ;
\end{gathered}
$$

and $d S$ denotes the surface element on $\rho(x)=t$. Then $L-k$ oscillates at $\partial G$.

Theorem 1 can easily be established by adapting the averaging procedures introduced in [7], [8], once we observe that near $\partial G$ we have by construction: $d x=(|\operatorname{grad} \rho|)^{-1} d S d \rho$.

Clearly Theorem 1 leads to upper bounds on $\mu$. To establish lower bounds we state:

THEOREM 2. Assume that for some $\beta$ and some positive function $f$, it follows that:

$$
c(x)-k \geqslant \sum D_{i}\left(a_{i j} D_{j} f\right) f^{-1}
$$

for all $x$ such that $\rho(x)>\beta$. Then $L-k$ is nonoscillatory at $\partial G$.

The proof of Theorem 2 is identical to the well-known one for the case of nonoscillation at $\infty$ (see, for example, [2]). Any suitable $f$ may be used in the theorem, but in view of our assumptions we consider $f=f(\rho)$.

COROLlaRY 1. Let $q$ be defined by:

$$
q(x)=\sum D_{i}\left(a_{i j} D_{j} \rho\right) / \sum a_{i j} D_{i} \rho D_{j} \rho,
$$


and assume that for $\rho(x)$ sufficiently large, $q(x)=p(\rho(x))$. Assume that $z(\rho)$ is such that the ordinary differential equation:

$$
-f^{\prime \prime}-p f^{\prime}+z f=0
$$

does not oscillate $($ at $\infty)$. If for $\rho(x)$ large:

$$
c(x)-k \geqslant\left(\sum a_{i j} D_{i} \rho D_{j} \rho\right)(z(\rho)),
$$

then $L-k$ is nonoscillatory at $\partial G$.

COROLlaRy 2. In addition to the hypothesis of Theorem 2 assume that $f^{\prime} \geqslant 0$ (resp. $\left.f^{\prime} \leqslant 0\right)$ near $\infty$. Then (2) may be replaced by:

$$
c(x)-k \geqslant \sum a_{i j} D_{i} \rho D_{j} \rho\left[\frac{f^{\prime \prime}+2 d f^{\prime}}{f}\right]
$$

where $2 d(t)=\sup _{\rho(x)=t} q(x)($ resp. inf $\operatorname{s(x)=t} q(x))$.

As an application of Theorem 2, Corollary 2 we state the following Kneser-type theorem.

THEOREM 3. Assume that for $\rho(x)$ large we have $\rho(x) q(x) \leqslant \frac{1}{2}$ (resp. $\left.\geqslant \frac{1}{2}\right)$. For $L-k$ to be nonoscillatory it is sufficient that:

$$
c(x)-k \geqslant\left\{-4^{-1} \rho^{-2}-d^{\prime}(\rho)-d^{2}(\rho)\right\} \sum a_{i j} D_{i} \rho D_{j} \rho,
$$

where $d$ is as defined above.

The proof follows from Corollary 2 once we observe that the equation

$$
-f^{\prime \prime}-2 d f^{\prime}+\left(-4^{-1} t^{-2}-d^{\prime}-d^{2}\right) f=0
$$

has solution $f(t)=\exp \left(-\int^{t} d\right) t^{+1 / 2}$. Observe that Theorem 3 contains Kneser's classical result. To allow for perturbations in Theorem 2 we state:

TheOREM 4. Let $\lambda_{0}(x)$ denote the least eigenvalue of $\left(a_{i j}(x)\right)$. Assume that $m>3$, and that for some $\beta, \varepsilon_{0}, \varepsilon$ where $\beta>0,0<\varepsilon_{0}<1,1<\varepsilon<1+2 / m$ :

$$
\begin{gathered}
{\left[\varepsilon_{0}\left(-\sum D_{i}\left(a_{i j} D_{j} f\right)\right) f^{-1}+c-k\right]_{-} \in L^{m /(2+m(1-\varepsilon))}((x \mid \rho>\beta)) ;} \\
\lambda_{0}^{-1} \in L^{1 /(\varepsilon-1)}((x \mid \rho>\beta)) .
\end{gathered}
$$

Then $L-k$ is nonoscillatory at $\partial G$.

The proof of Theorem 4 follows from an application of the theorem of Gagliardo-Nirenberg, similar to that used in [2].

We mention one other natural choice for $f$ in Theorem 2. Assume that $G=\cup_{\alpha>0}(x \mid s(x)=\alpha)$, for some regular function $s$. Here we do not require that $s(x)=\alpha$ be a closed surface. In such a case we may also choose $f=f(s)$ in Theorem 2 with $f(t)$ regular and positive for $t>0$. This approach has the disadvantage that neighbourhoods of $\partial G$ cannot in general be described in terms of the equipotentials of $s$, so that $f$ must be assumed regular and positive for $t>0$, as opposed to just for $t$ large. Whether this is a "better" choice than $f=f(\rho)$ will depend on the problem considered. 
To illustrate the above results we consider the following example where the calculations are simple. Let $G$ be the upper $\frac{1}{2}$ plane in $E^{2}$ and let $L$ be generated by the elliptic expression $l u=-\Delta u+c u$, with $c$ singular on parts of $x_{2}=0$, and regular otherwise. The simplest choice for $f$ in Theorem 2 is $f=x_{2}^{1 / 2}$, which implies that $L-k$ is nonoscillatory if $c-k \geqslant-4^{-1} x_{2}^{-2}$ in a neighbourhood of $\partial G$. To establish more nonoscillation criteria, as well as oscillation criteria, we choose $\rho\left(x_{1}, x_{2}\right)=\left(1+x_{1}^{2}+x_{2}^{2}\right) 2^{-1} x_{2}^{-1}$ (see also [11] where such a $\rho$ was used to extend the three circle theorem). A direct calculation shows that the right-hand side of (2) becomes:

$$
\left(\left(\rho^{2}-1\right) f^{\prime}\right)^{\prime} x_{2}^{-2} f^{-1}
$$

A simple variable change followed by a theorem of Hille [12, p. 56] shows that: $c-k \geqslant-x_{2}^{-2} z(\rho)$ in the exterior of some circle $\rho(x)=\alpha$, implies that $L-k$ is nonoscillatory as a consequence of Corollary 1 . Here we assume that $z>0, z$ is regular near $\infty$, and satisfies:

$$
\underset{\rho \rightarrow \infty}{\limsup }\left[-\rho\left\{\int_{\rho}^{\infty} z(t)\left(1-t^{2}\right)^{-1} d t\right\}\right]<4^{-1} .
$$

Proceeding in the other direction we find that (cf. Theorem 1):

$$
\begin{aligned}
& a(t)=2 \pi\left(t^{2}-1\right), \\
& b(t)=\left(t^{2}-1\right)^{-1 / 2} \int_{\rho(x)=t} x_{2}(c(x)-k) d S .
\end{aligned}
$$

We are therefore led to consider the equation:

$$
-\left(2 \pi\left(t^{2}-1\right) f^{\prime}\right)^{\prime}+b f=0
$$

A sufficient condition for this to oscillate is that, by Wintner's classical result $[12$, p. 70]:

$$
\int^{\infty} b(t)\left(t^{2}-1\right)^{-1} d t=-\infty
$$

That is:

$$
\lim _{\beta \rightarrow \infty}\left\{\int_{\beta>\rho>\alpha}(c(x)-k)\left(\rho(x)^{2}-1\right)^{-1} d x\right\}=-\infty .
$$

THEOREM 5. Let the above notation and assumptions hold. Assume that near $\partial G$ we have: $c(x) x_{2}^{-2} \geqslant-\frac{1}{4}$, and that (5) holds for any $k>0$. Then $\mu=0$, and $(-\infty, 0) \cap S(L)$ is finite.

Despite its special nature it does not seem possible to obtain Theorem 5 as a direct consequence of previously known nonoscillation results such as those obtained in [4], [6], [13], [14].

We conclude by observing that the constant $-\frac{1}{4}$ in Theorem 5 is optimal in the following sense: if $c(x) x_{2}^{-2}<-\delta / 4, \delta>1$, in a neighbourhood of $\partial G$ then $l$ is not bounded below, and therefore $\mu=-\infty$ for any selfadjoint extension of $l$. This can easily be seen by considering $\left(u_{n}, l u_{n}\right)$ with $1<\xi<\delta$, 
and:

$$
u_{n}(x)= \begin{cases}\sin \left(x_{1}\right) x_{2}^{1 / 2} \sin \left((\xi-1)^{1 / 2} 2^{-1} \ln \left(x_{2}\right)\right), & x \in R, \\ 0, & x \notin R,\end{cases}
$$

where $R$ is the rectangle $(0, \pi) \times\left(\exp \left(-2(n+1)(\xi-1)^{-1 / 2} \pi\right), \exp (-2 n(\xi-\right.$ 1) $-1 / 2 \pi)$ ).

\section{REFERENCES}

1. W. Allegretto, On the equivalence of two types of nonoscillation for elliptic operators, Pacific J. Math. 55 (1974), 319-328. MR 51 \# 10827.

2. Nonoscillation criteria for elliptic equations in conical domains, Proc. Amer. Math. Soc. 63 (1977), 245-250.

3. I. M. Glazman, Direct methods of qualitative spectral analysis of singular differential operators, Israel Program for Scientific Translations, Davey, New York, 1965. MR 32 \#2938.

4. V. Headley and C. A. Swanson, Oscillation criteria for elliptic equations, Pacific J. Math. 27 (1968), 501-506. MR 38 \# 4797.

5. K. Kreith, Oscillation theory, Lecture Notes in Math., vol. 324, Springer-Verlag, Berlin, 1973.

6. , Oscillation theorems for elliptic equations, Proc. Amer. Math. Soc. 15 (1964), 341-344. MR 33 \#6098.

7. K. Kreith and C. Travis, Oscillation criteria for self-adjoint elliptic differential equations, Pacific J. Math. 41 (1972), 743-753. MR 47 \# 7189.

8. E. Noussair, Elliptic equations of order $2 m$, J. Differential Equations 10 (1971), 100-111. MR 43 \#6564.

9. J. Piepenbrink, Nonoscillatory elliptic equations, J. Differential Equations 15 (1974), 541550. MR 49 \#7573.

10. __ A conjecture of Glazman, J. Differential Equations 24 (1977), 173-177.

11. M. Protter and H. Weinberger, Maximum principles in differential equations, Prentice-Hall, Englewood Cliffs, N. J., 1967. MR 36 \#2935.

12. C. A. Swanson, Comparison and oscillation theory of linear differential equations, Academic Press, New York, 1968.

13. , Nonoscillation criteria for elliptic equations, Canad. Math. Bull. 12 (1969), 275-280. MR 41 \#621.

14. N. Yoshida, Nonoscillation of elliptic equations of second order, Hiroshima Math. J. 4 (1974), 279-284. MR 50 \#743.

INSTITUTE OF MAThEMATICS, UNIVERSITY OF FLORENCE, FLORENCE, ITALY

Current address: Department of Mathematics, University of Alberta, Edmonton, Alberta, Canada T6G 2G1 